\title{
Miliary Nodularity in a Patient Receiving TNF Inhibitors Is Not Always Miliary Tuberculosis
}

MUHAMMAD HAROON, MB, MMedSc, MRCPI; BREDA CUSHEN, MRCPI; DOUGLAS VEALE, MD, FRCPI, FRCP, Professor, Department of Rheumatology, Bone and Joint Unit, St. Vincent's University Hospital, Dublin, Ireland. Address correspondence to Dr. Haroon;

E-mail: mharoon301@hotmail.com. J Rheumatol 2012;39:651-2; doi:10.3899/jrheum.111169

Malignancy can be common in patients with rheumatoid arthritis (RA) receiving tumor necrosis factor (TNF) inhibitors, and a tissue analysis remains crucial in establishing diagnosis.

A 61-year-old woman, diagnosed with seropositive RA (rheumatoid factor and anticitrullinated protein antibody) in 2009, was in remission under treatment with etanercept and methotrexate. She was an ex-smoker. She presented with a 5-month history of dry, irritating cough, which was associ- ated with pleuritic chest pains and exertional dyspnea. Examination revealed normal vital signs and oxygen saturation was $95 \%$ on room air. Laboratory testing was unremarkable, apart from mild leukocytosis and elevated inflammatory markers. Chest radiograph revealed collapse and consolidation of the right upper lobe with widespread miliary nodularity. Given the radiographic findings and the risk of reactivation of tuberculosis (TB) with TNF inhibitors, a provisional diagnosis of miliary TB was made and she was
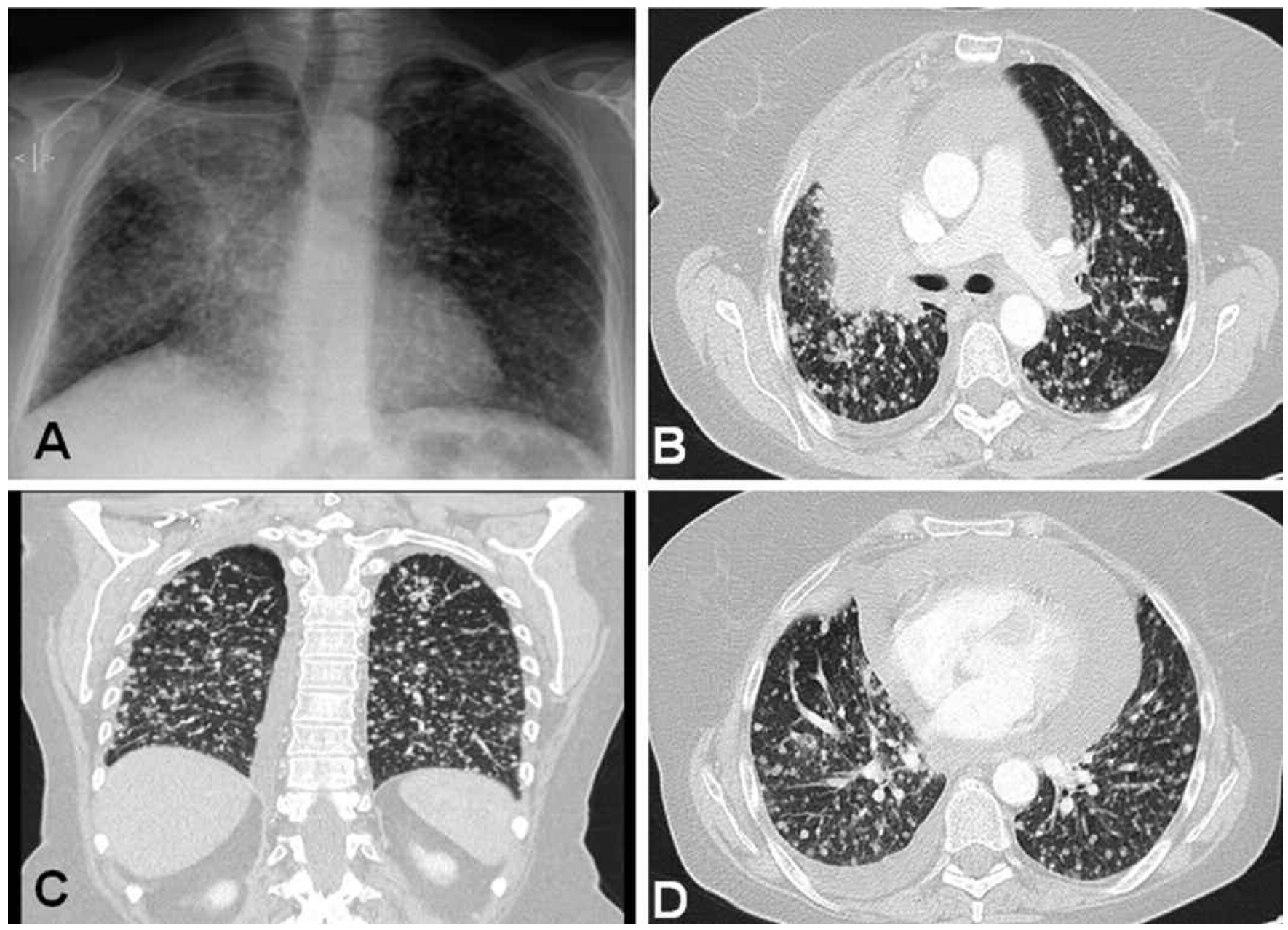

Figure 1. A. Chest radiograph reveals widespread miliary nodularity in both lungs along with biapical pleural thickening, which raised the suspicion of miliary tuberculosis. B to D. Several slices of the computed tomography scan of thorax showing innumerable randomly distributed pulmonary nodules throughout both lungs ranging in size from 0.1 to $0.7 \mathrm{~cm}$, which are noncalcified and noncavitating. 
started on quadruple therapy. Three subsequent sputum samples were negative for acid-fast bacilli, as was her Mantoux tuberculin skin test. Computed tomography scan of thorax showed innumerable randomly distributed pulmonary nodules throughout both lungs ranging in size from 0.1 to $0.7 \mathrm{~cm}$. The right upper lobe was collapsed with luminal narrowing of the bronchus to the right upper lobe, but no endobronchial mass was identified. Endobronchial examination and biopsy showed a moderately differentiated adenocarcinoma within lymphatic spaces, staining positive for thyroid transcription factor-1, consistent with a primary lung cancer. She was diagnosed with lymphangitic carcinomatosis and stage 4 primary lung adenocarcinoma. Her respiratory condition deteriorated rapidly and she died within a week of admission.

Although pattern recognition is an important tool of the physician, caution and thinking outside the box is prudent. Since malignancy can be common in patients with RA receiving TNF inhibitor, every effort should be made to obtain a tissue analysis, which remains crucial in establishing diagnosis and guiding therapy. 